\title{
Automatic flight control requirements for transition flight phases when converting long endurance fixed wing UAV to VTOL aircraft
}

\begin{abstract}
This paper reviews the 2 most critical flight phases for a Long Endurance Engine Powered Fixed-Wing Hybrid UAV - the transition from hover to forward flight and visa-versa. These phases of flight are necessary as the consequences of replacing the unsuitable horizontal takeoff and landing ability with the more convenient vertical take-off and landing capability. After a brief description on the flight profile and a proposal for the hybrid configuration, the paper suggests 3 strategies to enable the hybrid fixed-wing UAV to transition from hover to forward flight and 1 strategy to transition from forward flight to hover. These strategies present themselves as part of the fundamental requirements for the onboard automatic flight control system responsible to regulate these 2 flight phases.
\end{abstract}

Keyword: Hybrid UAV; Quadrotor; Drones; VTOL UAV; VTOL transition speeds; Precision agriculture; Crop dusting 